OPEN ACCESS

Edited by:

Itamar Ronen,

Leiden University Medical Center,

Netherlands

Reviewed by:

Donnie Cameron,

University of East Anglia,

United Kingdom

Artur Krzyżak,

AGH University of Science

and Technology, Poland

${ }^{*}$ Correspondence:

Felix T. Kurz

felix.kurz@med.uni-heidelberg.de;

felix.kurz@gmx.net

Specialty section: This article was submitted to

Brain Imaging Methods,

a section of the journal

Frontiers in Neuroscience

Received: 16 December 2020

Accepted: 10 February 2021

Published: 03 March 2021

Citation:

Jende JME, Kender Z,

Mooshage C, Groener JB,

Alvarez-Ramos L, Kollmer J, Juerchott A, Hahn A, Heiland S,

Nawroth P, Bendszus M, Kopf S and

Kurz FT (2021) Diffusion Tensor Imaging of the Sciatic Nerve as

a Surrogate Marker for Nerve Functionality of the Upper and Lower Limb in Patients With Diabetes and Prediabetes.

Front. Neurosci. 15:642589. doi: 10.3389/fnins.2021.642589

\section{Diffusion Tensor Imaging of the} Sciatic Nerve as a Surrogate Marker for Nerve Functionality of the Upper and Lower Limb in Patients With Diabetes and Prediabetes

\author{
Johann M. E. Jende ${ }^{1}$, Zoltan Kender ${ }^{2}$, Christoph Mooshage ${ }^{1}$, Jan B. Groener ${ }^{2,3,4}$, \\ Lucia Alvarez-Ramos ${ }^{2}$, Jennifer Kollmer ${ }^{1}$, Alexander Juerchott ${ }^{1}$, Artur Hahn ${ }^{1}$, \\ Sabine Heiland ${ }^{1,5}$, Peter Nawroth ${ }^{2,4,6}$, Martin Bendszus ${ }^{1}$, Stefan Kopf ${ }^{2,4}$ and \\ Felix T. Kurz ${ }^{1 *}$ \\ ${ }^{1}$ Department of Neuroradiology, Heidelberg University Hospital, Heidelberg, Germany, ${ }^{2}$ Department of Endocrinology, \\ Diabetology and Clinical Chemistry, Heidelberg University Hospital, Heidelberg, Germany, ${ }^{3}$ Medicover Neuroendocrinology, \\ Munich, Germany, ${ }^{4}$ German Center of Diabetes Research (DZD), Associated Partner in the DZD, München-Neuherberg, \\ Germany, ${ }^{5}$ Division of Experimental Radiology, Department of Neuroradiology, Heidelberg, Germany, ${ }^{6}$ Joint Institute \\ for Diabetes and Cancer at Helmholtz-Zentrum Munich and Heidelberg University, Heidelberg, Germany
}

Background: Nerve damage in diabetic neuropathy (DN) is assumed to begin in the distal legs with a subsequent progression to hands and arms at later stages. In contrast, recent studies have found that lower limb nerve lesions in DN predominate at the proximal sciatic nerve and that, in the upper limb, nerve functions can be impaired at early stages of DN.

Materials and Methods: In this prospective, single-center cross-sectional study, participants underwent diffusion-weighted 3 Tesla magnetic resonance neurography in order to calculate the sciatic nerve's fractional anisotropy (FA), a surrogate parameter for structural nerve integrity. Results were correlated with clinical and electrophysiological assessments of the lower limb and an examination of hand function derived from the Purdue Pegboard Test.

Results: Overall, 71 patients with diabetes, 11 patients with prediabetes and 25 agematched control subjects took part in this study. In patients with diabetes, the sciatic nerve's FA showed positive correlations with tibial and peroneal nerve conduction velocities ( $r=0.62 ; p<0.001$ and $r=0.56 ; p<0.001$, respectively), and tibial and peroneal nerve compound motor action potentials $(r=0.62 ; p<0.001$ and $r=0.63$; $p<0.001$, respectively). Moreover, the sciatic nerve's FA was correlated with the Pegboard Test results in patients with diabetes $(r=0.52 ; p<0.001)$, prediabetes $(r=0.76 ; p<0.001)$ and in controls $(r=0.79 ; p=0.007)$. 
Conclusion: This study is the first to show that the sciatic nerve's FA is a surrogate marker for functional and electrophysiological parameters of both upper and lower limbs in patients with diabetes and prediabetes, suggesting that nerve damage in these patients is not restricted to the level of the symptomatic limbs but rather affects the entire peripheral nervous system.

Keywords: magnetic resonance imaging, diabetic polyneuropathy, magnetic resonance neurography, diffusion tensor imaging, fractional anisotropy, diabetes, prediabetes

\section{INTRODUCTION}

Distal symmetric diabetic polyneuropathy (DN) is one of the most severe complications of diabetes mellitus (Tesfaye et al., 2005; Feldman et al., 2017). DN is generally acknowledged to be a late complication of diabetes that starts at the level of the feet and then progresses further upwards until, at later stages, the upper limbs become involved as well, starting at the level of the hands (Nawroth et al., 2018). In contrast to the progression of clinical symptoms, recent studies applying high-resolution magnetic resonance neurography (MRN) at three Tesla (3T) have come to show that nerve lesions predominate proximally at the level of the sciatic nerve and that the sciatic nerve's fractional anisotropy (FA), a dimensionless quantity for directed diffusion in nerve tissue, is a highly sensitive parameter for structural nerve damage in patients with diabetic neuropathy in previous clinical studies (Vaeggemose et al., 2017b; Jende et al., 2019, 2020a). Despite the assumption that length-dependent nerve damage in DN starts at the level of the feet and progresses to further proximally with an involvement of the upper limbs at later stages, recent studies revealed that sensory and motor functions of the upper limb are frequently affected already at early stages of DN but often remain undiagnosed until a certain degree of functional impairment becomes apparent, indicating that the progression of nerve fiber damage at the level of the hands and arms may parallel the progression of nerve fiber damage at the level of the feet and legs (Kopf et al., 2018a; Yang et al., 2018).

The aim of this study was to elucidate correlations of the sciatic nerve's FA with clinical and electrophysiological parameters of the upper and lower limbs in patients with prediabetes and diabetes, and in an age-matched control group. Therefore, we chose an MRN protocol that combined T2-weighted and diffusionweighted sequences with a subsequent automated approach for the calculation of the sciatic nerve's FA.

\section{MATERIALS AND METHODS}

\section{Study Design and Participants}

The local ethics committee approved this study (HEIST-DiC, local ethics number S-383/2016

Abbreviations: CI, confidence interval; CMAP, compound motor action potential; $\mathrm{DN}$, diabetic neuropathy; $\mathrm{mV}$, microvolt; $\mathrm{NCV}$, nerve conduction velocity; NDS, neuropathy disability score; NSS, neuropathy severity score; SNAP, sensory nerve action potential.

${ }^{1}$ clinicaltrials.gov all study participants gave informed, written consent. Prediabetes was defined as fasting glucose levels of $100-125 \mathrm{md} / \mathrm{dL}$ or a 2-h glucose level of 140-199 mg/dL after $75 \mathrm{~g}$ glucose intake. Participants with glucose levels below these limits were defined as normal (healthy controls). Participants with glucose levels above the limit and absence of GAD or IA2 antibodies were defined as newly diagnosed type 2 diabetes (Kopf et al., 2018b). Overall, 71 patients with diabetes (12 with type 1 diabetes, 59 with type 2 diabetes; 26 female, 45 male; mean age $61.07 \pm 1.57$; age range 21-84 years), 11 patients with prediabetes (6 female, 5 male, $61.82 \pm 2.85$, age range 47-78) and 25 age-matched control subjects ( 16 female, 9 male, mean age $56.24 \pm 2.17$, age range 30-77) took part in this study between June 2016 and May 2020. Study participants were recruited from the Outpatient Clinic of Internal Medicine at Heidelberg University Hospital (Z.K., J.B.G., L.A., and S.K.). Participants formed a random series, meaning that they were not recruited and examined groupwise but in a randomized order. All participants were right-handed. Overall exclusion criteria were age $<18$, pregnancy, any history of lumbar surgery or disk extrusion, any contraindications for MR imaging, any other neuropathy-associated risk factors such as alcohol abuse, hypovitaminosis, malignant or infectious diseases, any previous or ongoing exposure to neurotoxic agents, monoclonal gammopathy, and any chronic neurological diseases such as Parkinson's disease, multiple sclerosis, or restless legs syndrome. The process of patient recruitment is illustrated in Figure 1.

\section{Clinical and Electrophysiologic Examination}

A detailed medical history was documented for every participant. The examination of neuropathic symptoms was performed according to the guidelines of the German Society for Diabetology comprising the neuropathy symptom score (NSS), the Neuropathy Disability Score (NDS) and electrophysiological examinations as outlined below (Young et al., 1993).

The electrophysiological examination (Viasys Healthcare VikingQuest ${ }^{\circledR}$, Viasys Healthcare GmbH, Höchberg) of the right leg included: distal motor latencies of the right tibial and peroneal nerve, motor and sensory amplitudes (CMAPs and SNAPs, respectively) of the tibial, peroneal and sural nerves and nerve conduction velocities (NCVs) of the tibial, peroneal and sural nerves. It was assured that skin temperature was at least $32^{\circ} \mathrm{C}$ throughout the examination. Blood was drawn in fasting state and processed immediately under standardized conditions in the central laboratory of Heidelberg University 


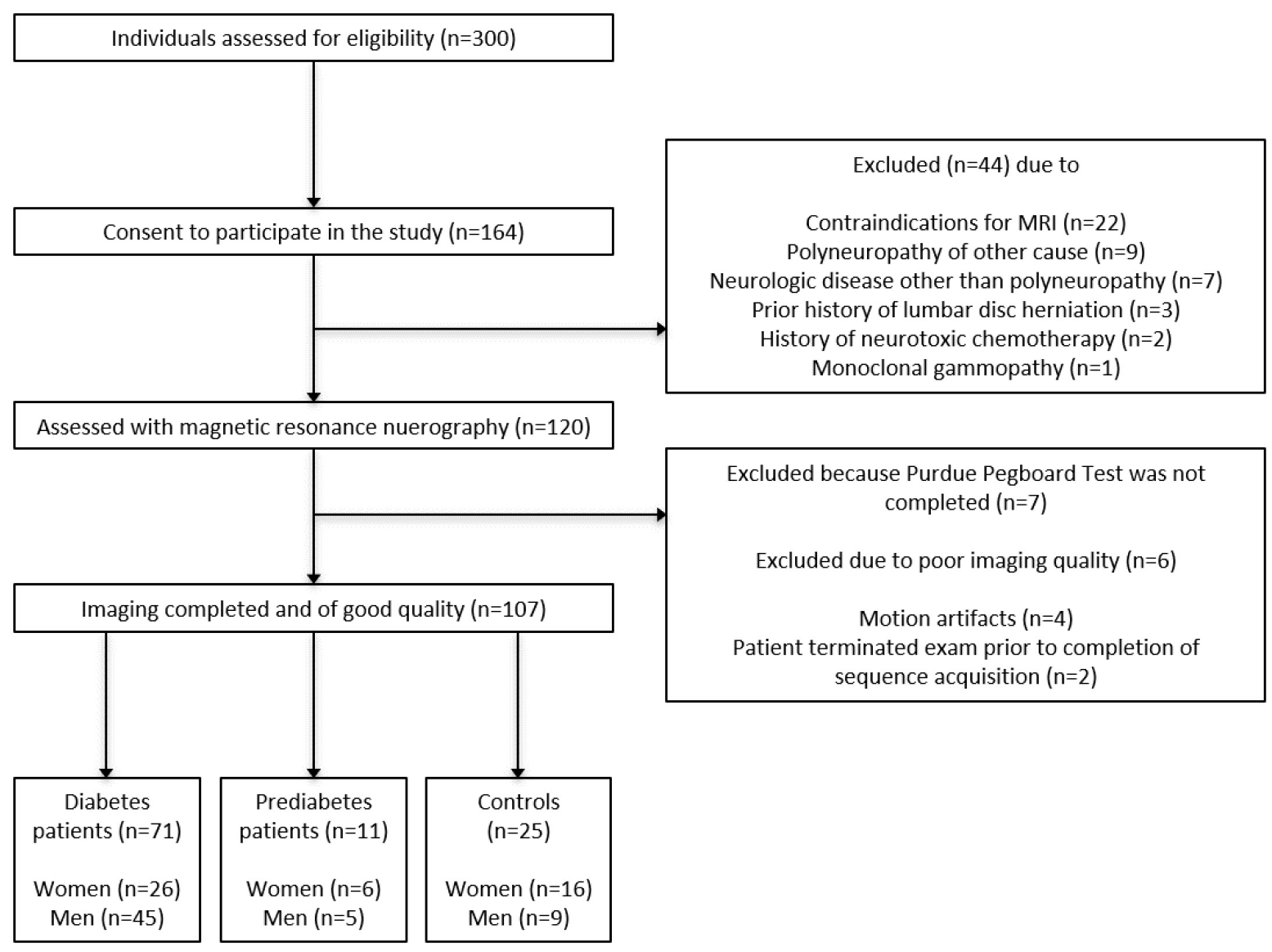

FIGURE 1 | Process of patient recruitment.

Hospital. All examinations were performed by clinicians with expertise in diabetology and more than 8 years of clinical experience (Z.K., J.B.G., and S.K.) and were surveyed by a clinician with expertise in internal medicine, diabetology, and laboratory medicine (P.N.) with more than 30 years of experience.

\section{Measurement of Hand Dexterity and Fine Motoric Skills by Using the Purdue Pegboard Test}

The Purdue Pegboard Test (Model 32020; Lafayette Instrument Co., Lafayette, IN, United States), an established and commonly used test for the assessment of both gross and fine motor skills of the arms and hands, was used to assess motoric skills and manual dexterity (Symonds et al., 2017). The test employs a board with two parallel rows of 25 cavities into which cylindrical metal pegs are to be placed and comprises a total of four trials. The test includes four subtests: testing of the dominant hand, testing of the non-dominant hand, testing of both hands, and an assembly test allowing use of both hands as described before. In the subsets for preferred, non-preferred, and both hands the examinee is required to place the pins as fast as possible, the score being the number of pins placed after $30 \mathrm{~s}$ (O’Donnell et al., 2017).

\section{MRN Imaging Protocol}

We performed high-resolution MRN of the right thigh in a 3.0 Tesla MR-scanner (Magnetom TIM-TRIO, Siemens Healthineers, Erlangen, Germany) for all study participants, using a 15-channel transmit-receive extremity coil. Participants were put in a supine position on the MRI transport table with the legs facing the scanner opening; the leg imaging coil, a 15channel transit-receive extremity coil, was placed around the right midthigh and mid lower leg. The following sequences were used:

(1) Axial high resolution T2-weighted turbo spin echo (TSE) 2D sequence with spectral fat saturation (mode: strong) without water suppression or magnetization preparation. The following parameters were used: relaxation time $(\mathrm{TR})=5,970 \mathrm{~ms}$, echo time $(\mathrm{TE})=55 \mathrm{~ms}$, field of view $($ FOV $)=160 \mathrm{~mm}^{2} \times 160 \mathrm{~mm}^{2}$, matrix size $=512 \times 512$, slice thickness $=4 \mathrm{~mm}$, interslice gap $=0.8 \mathrm{~mm}$, voxel size $=0.3 \mathrm{~mm}^{3} \times 0.3 \mathrm{~mm}^{3} \times 4.0 \mathrm{~mm}^{3}, 24$ slices, 24 acquired images, receiver bandwidth $=181 \mathrm{~Hz} /$ pixel, echo spacing $=11.1 \mathrm{~ms}$, turbo factor $=13,15$ echo trains per slice, parallel imaging factor $=2$, averages $=3$, acquisition time $=4: 42 \mathrm{~min}$. 
(2) Axial diffusion-weighted 2-dimensional echo-planar sequence images were recorded with spectral attenuated inversion recovery fat suppression (saturation mode: skewed). The following parameters were used: $\mathrm{TR}=5,100 \mathrm{~ms} ; \mathrm{TE}=92.8 \mathrm{~ms} ; \mathrm{b}=0$ and $1,000 \mathrm{~s} / \mathrm{mm}^{2}$; directions $=20$; field of view $160 \mathrm{~mm}^{2} \times 160 \mathrm{~mm}^{2}$; matrix size $128 \times 128$; axial fat-suppressed, diffusion-weighted 2-dimensional echo-planar sequence with the following parameters: $\mathrm{TR}=5,100 \mathrm{~ms} ; \mathrm{TE}=92.8 \mathrm{~ms} ; \mathrm{b}=0$ and $1,000 \mathrm{~s} / \mathrm{mm}^{2}$; directions $=20$; field of view $=160 \mathrm{~mm}^{2} \times 160 \mathrm{~mm}^{2}$; matrix size $=128 \times 128 ;$ slice thickness $=4 \mathrm{~mm}$; voxel size $=1.3 \mathrm{~mm}^{3} \times 1.3 \mathrm{~mm}^{3} \times 4 \mathrm{~mm}^{3}$; no interslice gap, 24 slices, 1,512 acquired images, receiver bandwidth $=1,396 \mathrm{~Hz} /$ pixel, EPI factor $=128$, parallel imaging factor $=3$, averages $=3$, acquisition time $=5: 47 \mathrm{~min}$.

MRN sequences were centered on the sciatic nerve's bifurcation to ascertain that the anatomical region mapped by MRN was comparable in all participants. All MRN Studies were performed by radiologists with more than 5 years of clinical experiences in MRN studies (J.M.E.J., J.K., A.J., and F.T.K.).

\section{Image Post-processing and Analysis}

The MRN analysis followed an established analysis pipeline for diffusion tensor imaging (DTI) images (Christidi et al., 2016). All images were pseudonymized and analyzed in an automated approach (J.M.E.J., C.M., F.T.K.) using Nordic BRAINEX, a Food and Drug Administration (FDA) approved processing software designed for automated calculation and reconstruction of fiber tracts in diffusion weighted imaging. Nordic BrainEx uses a variant of the Fiber Assignment by Continuous Tracking (FACT) algorithm to obtain fiber tracking within a volume-of-interest (VOI) (Mori et al., 1999; NordicNeuroLab AS, 2019). We closely followed the DTI module instructions for Nordic BRAINEX (NordicNeuroLab AS, 2019). DTI setting configurations were extracted from the headers of the uploaded DTI image data (e.g., number of gradient directions, averages, etc.). First, we co-registered DTI images to the T2w images. For subsequent preprocessing, we chose the options "motion correction" and "Eddy current correction" and adjusted a noise threshold to include all voxels within the sciatic nerve. We further chose a FA cutoff $>0.1$ as in previous studies on DTI analyses in peripheral nerves (Kwon et al., 2015; Oudeman et al., 2020) to reduce the impact of intra- and perineural fat on the calculated FA of fascicular structures (Kwon et al., 2015; Oudeman et al., 2020). The tract turning angle was chosen as 41.4 degrees, the minimum fiber length at $20 \mathrm{~mm}$, and the number of seeds per voxel as 1 , see also (NordicNeuroLab AS, 2019). We then manually focused a cube shaped VOI on the sciatic nerve.

The tensor eigenvalues $\lambda_{1}, \lambda_{2}$, and $\lambda_{3}$ and the average FA of the segmented nerve fibers were automatically determined in Nordic BRAINEX (NordicNeuroLab AS, 2019). Axial diffusivity (AD), radial diffusivity (RD) and mean diffusivity (MD) were calculated based on the obtained tensor eigenvalues as $\mathrm{AD}=\lambda_{1}$, $\mathrm{RD}=\left(\lambda_{2}+\lambda_{3}\right) / 2$, and $\mathrm{MD}=\left(\lambda_{1}+\lambda_{2}+\lambda_{3}\right) / 3{ }^{16}$. A graphic overview of the process of image co-registration and nerve segmentation is given in Figure 2.

\section{Statistical Analysis}

Statistical data analysis was performed with GraphPad Prism 7 and Matlab v7.14.0.0739, R2012a (J.M.E.J., A.H., and F.T.K.). We tested for Gaussian normal distribution with the D'Agostino-Pearson omnibus normality test. Depending on Gaussian distribution, ANOVAs, or Kruskal-Wallis tests were performed for comparisons of three groups and Bonferonicorrected Spearman or Pearson correlation coefficients for correlation analysis.

For all tests, the level of significance was defined at $p<0.05$. All results are presented as mean values \pm standard deviation.

\section{RESULTS}

\section{MRN Imaging, Demographic, Clinical and Serological Data}

There was no difference between the three groups for participants' age $(p=0.231)$. HbAlc levels and triglyceride levels were higher in patients with diabetes when compared to patients with prediabetes or controls $(p<0.001$ and $p=0.002$, respectively). The total Pegboard score and the sciatic nerve's FA were lower in patients with diabetes when compared to controls $(p=0.006)$. No such difference could be found for AD $(p=0.147)$, $\mathrm{RD}(p=0.179)$, or $\mathrm{MD}(p=0.810)$. In controls, patients with prediabetes and patients with diabetes, the total pegboard score was negatively correlated with age $(r=-0.64 ; p=0.001, r=0.82$; $p=0.002$, and $r=0.60 ; p<0.001$, respectively). The NDS and the NSS score were higher in patients with diabetes when compared to controls $(p<0.001)$. The mean FA for controls $(0.48 \pm 0.06)$ and prediabetic patients $(0.48 \pm 0.08)$ was well within the reference range of a healthy group of volunteers $[0.507 \pm 0.05$; (Kronlage et al., 2018)], whereas the mean FA for patients with diabetes was not $(0.43 \pm 0.08)$. An overview of participants' characteristics is given in Table 1. Representative sciatic nerve fiber tracts from individuals of the three groups of participants (diabetes, prediabetes, controls) can be found in Figure 3. It can be seen that fiber tracts in the control patient are dense and contiguous (Figure 3a), which decreases for the prediabetes and diabetes patients (Figures $\mathbf{3 b}, \mathbf{c}$ ).

A negative association was found between the sciatic nerves' FA and patients' age in patients with diabetes $(r=-0.48$; $p<0.001)$ and controls $(r=-0.63 ; p=0.001)$ but not in patients with prediabetes $(r=0.44 ; p=0.202)$. FA was negatively correlated with the NDS score in patients with diabetes, $(r=-0.53 ; p<0.001)$ and controls $(r=-0.47$; $p=0.017)$. A similar, yet not significant trend was seen in patients with prediabetes $(r=-0.58 ; p=0.082)$. In patients with diabetes, further negative associations of the FA were found with cystatin $\mathrm{C}$ $(r=-0.45 ; p<0.001)$. A detailed survey of correlations between the sciatic nerve's FA and demographic, clinical, and serological parameters is given in Table 2.

Similarly, as in previous studies, (Vaeggemose et al., 2017a, 2020; Kronlage et al., 2018). RD and MD (or apparent diffusion 


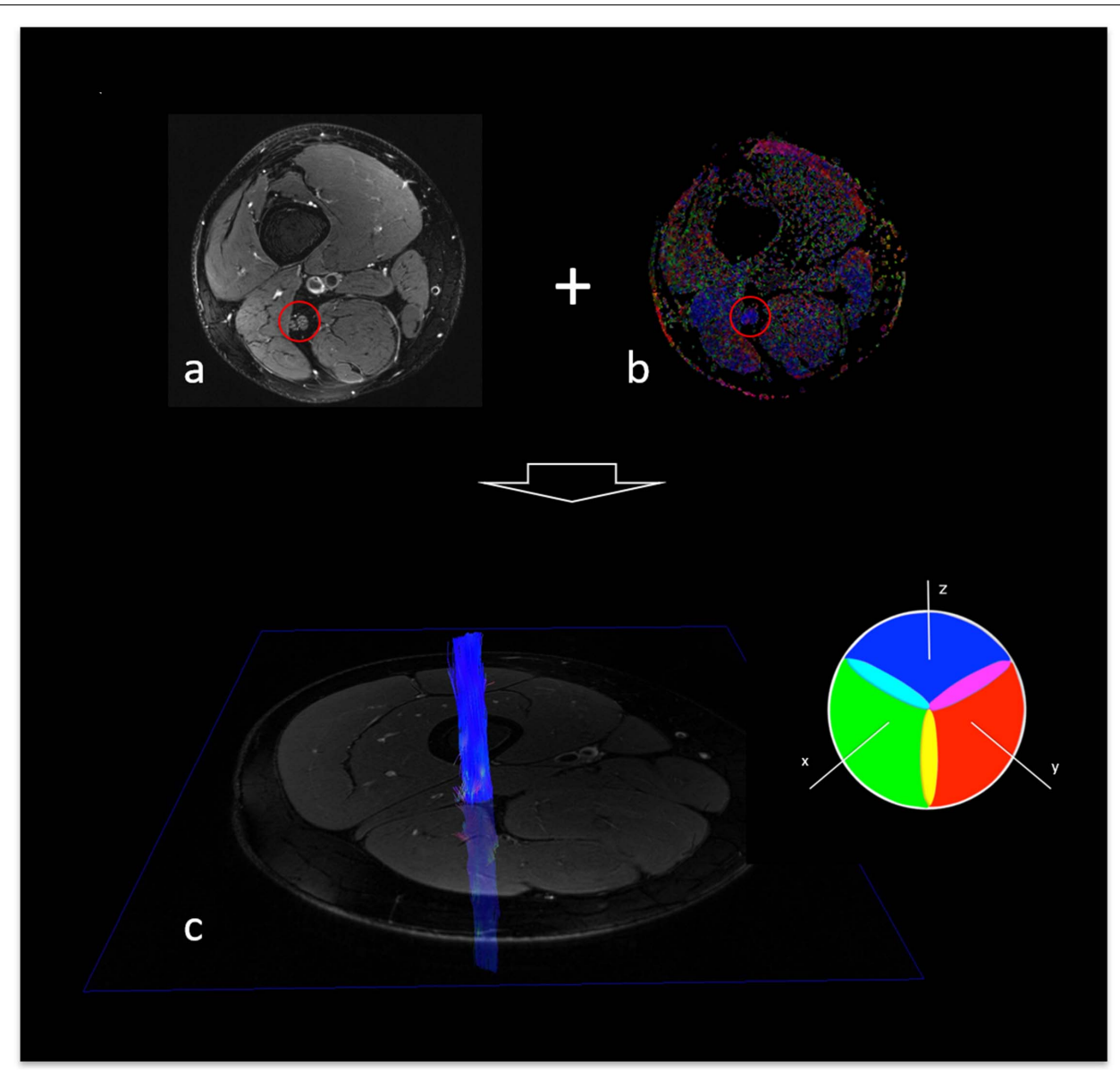

FIGURE 2 | Fiber tracking of the sciatic nerve. (a) T2-weighted, fat suppressed image of the right thigh, depicting the sciatic nerve (red circle). (b) Colored map of fractional anisotropy (sciatic nerve encirceled in red). (c) Reconstructed, 3-dimensional fiber track of the right sciatic nerve with color encoding according to the DTI eigenvector color map where voxel color reflects the direction of the diffusion tensor in that voxel. Color intensity is scaled with the FA.

coefficient ADC) showed correlations with clinical parameters that are in line with the correlations obtained for the fractional anisotropy FA. For instance, the mean diffusivity in the diabetes cohort is highly correlated with tibial NCV $(r=-0.31$, $p=0.004)$, and the Pegboard of the dominant and non-dominant hand $(r=-0.26, p=0.026$, and $r=-0.31, p=0.008$, respectively), as is the FA $(r=0.47, p<0.001$, and $r=0.61$, $p<0.001$, and $r=0.43, p<0.001$, respectively). Correlations of FA values with clinical, epidemiological, and serological data were generally better than those of $\mathrm{RD}$ and $\mathrm{MD}$, which is why we focused on FA values; however, correlations of $\mathrm{AD}$, $\mathrm{RD}, \mathrm{MD}$, and DTI tensor eigenvalues $\lambda_{2}$ and $\lambda_{3}$ with all relevant parameters are given in the Supplementary Tables 1-5, respectively.

\section{MRN Results and Electrophysiological Data of the Lower Limb}

In patients with diabetes, the sciatic nerves' FA showed positive correlations with tibial and peroneal NCVs $(r=0.47 ; p<0.001$, $r=0.52 ; p<0.001$, respectively), with tibial and peroneal nerve compound muscle action potential (CMAP) amplitudes and sural nerve sensory nerve action potential (SNAP) amplitudes $(r=0.62 ; p<0.001, r=0.62 ; p<0.001$, and $r=0.47$; $p=0.006$, respectively). A similar, yet not significant trend was found for tibial nerve NCV and CMAP amplitudes in patients with prediabetes $(r=0.61 ; p=0.081$ and $r=0.61 ; p=0.081$, respectively). A summary of all correlations between the FA and electrophysiological data of the lower limb is given in Table 2.

\section{MRN Results and Hand Function}

The sciatic nerves' FA correlated positively with the total score of the pegboard-test in patients with diabetes, patients with prediabetes and controls $(r=0.52 ; p<0.001, r=0.79 ; p=0.007$, and $r=0.76 ; p<0.001$, respectively, Figures 4A-C). In all 4 subtests that comprise the Pegboard test, this finding could be reproduced: for patients with diabetes, patients with prediabetes and controls, significant correlations were found between FA and Pegboard scores of the dominant hand (Figures 4D-F) nondominant hand (Figures 4G-I), testing of both hands (Figures 4J$\mathbf{L}$ ), and the assembly test (Figures $4 \mathbf{M}-\mathbf{O}$ ). A summary of the correlations between the sciatic nerves' FA and results of the Purdue Pegboard test is given in Table 2. 
TABLE 1 | Demographic, clinical, serological, and electrophysiological data of patients with diabetes, patients with prediabetes, and controls.

\begin{tabular}{|c|c|c|c|c|}
\hline & Diabetes patients & Prediabetes patients & Controls & $p$ \\
\hline Age (years) & $61.1 \pm 13.5$ & $61.8 \pm 9.5$ & $56.2 \pm 10.9$ & 0.231 \\
\hline Number of women & 26 & 6 & 16 & n.a. \\
\hline Number of men & 45 & 5 & 9 & n.a. \\
\hline Body mass index $\left(\mathrm{kg} / \mathrm{m}^{2}\right)$ & $28.8 \pm 4.1$ & $28.5 \pm 5.8$ & $26.7 \pm 6.4$ & 0.131 \\
\hline NDS & $3.0 \pm 2.9$ & $1.1 \pm 1.7$ & $0.9 \pm 1.3$ & $<0.001$ \\
\hline NSS & $3.5 \pm 3.3$ & $1.9 \pm 2.9$ & $0.1 \pm 0.6$ & $<0.001$ \\
\hline Sciatic nerve's FA & $0.43 \pm 0.08$ & $0.48 \pm 0.08$ & $0.48 \pm 0.06$ & 0.006 \\
\hline Total purdue pegboard score & $60.8 \pm 11.8$ & $65.3 \pm 11.7$ & $71.6 \pm 11.2$ & $<0.001$ \\
\hline \multirow[t]{2}{*}{$\mathrm{HbA} 1 \mathrm{c}[\%(\mathrm{mmol} / \mathrm{mol})]$} & $7.2 \pm 1.28$ & $5.7 \pm 0.48$ & $5.3 \pm 0.36$ & $<0.001$ \\
\hline & $(55 \pm 14)$ & $(39 \pm 5)$ & $(34 \pm 4)$ & \\
\hline Cystatin C (mg/dl) & $0.96 \pm 0.25$ & $0.98 \pm 0.19$ & $0.89 \pm 0.14$ & 0.451 \\
\hline GFR (ml/min) & $88.6 \pm 18.6$ & $84.7 \pm 17.3$ & $89.9 \pm 15.9$ & 0.660 \\
\hline Triglycerides (mg/dl) & $175.1 \pm 187.0$ & $145.4 \pm 96.8$ & $101.6 \pm 44.0$ & 0.002 \\
\hline Total Cholesterol (mg/dl) & $188.7 \pm 43.4$ & $197.3 \pm 41.8$ & $209.0 \pm 40.0$ & 0.056 \\
\hline HDL Cholesterol & $53.9 \pm 19.1$ & $54.4 \pm 14.7$ & $62.5 \pm 19.0$ & 0.033 \\
\hline LDL Cholesterol & $104.2 \pm 34.5$ & $113.8 \pm 41.2$ & $126.2 \pm 36.7$ & 0.007 \\
\hline Sural NCV (m/s) & $44.0 \pm 11.5$ & $45.0 \pm 5.5$ & $45.6 \pm 8.6$ & 0.882 \\
\hline Sural SNAP $(\mu \mathrm{V})$ & $6.3 \pm 4.2$ & $6.2 \pm 3.5$ & $8.4 \pm 5.8$ & 0.236 \\
\hline Peroneal NCV (m/s) & $40.1 \pm 8.2$ & $42.6 \pm 3.3$ & $45.4 \pm 3.9$ & 0.002 \\
\hline Peroneal CMAP $(\mu \mathrm{V})$ & $6.1 \pm 3.9$ & $5.8 \pm 3.2$ & $8.6 \pm 4.9$ & 0.033 \\
\hline Tibial NCV (m/s) & $40.3 \pm 8.0$ & $44.4 \pm 2.2$ & $46.5 \pm 5.1$ & $<0.001$ \\
\hline Tibial CMAP $(\mu \bigvee)$ & $11.3 \pm 8.8$ & $13.2 \pm 6.2$ & $15.4 \pm 6.5$ & 0.033 \\
\hline
\end{tabular}

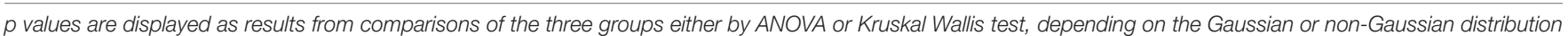

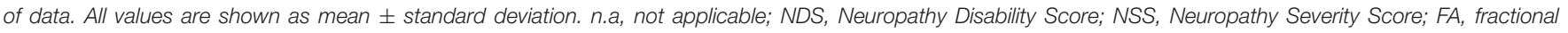

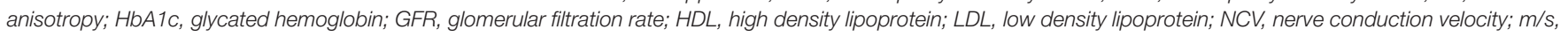
meters per second; SNAP, sensory nerve action potential; CMAP, compound motor action potential; $\mu \mathrm{V}$, microvolt.

Corresponding correlations for the subgroups of men and women are clearly visible, especially for the subgroup of women. We also tabularized the results from correlation analyses in both subgroups in Supplementary Table 6. Except for the Pegboard test of both hands in the men subgroup, all Pegboard parameters were significantly correlated with FA in the diabetes group. There was also a mild correlation of the Pegboard test of the dominant hand in the women subgroup and the control subgroup, as well as some further significant correlations between Pegboard parameters and FA in the control subgroups. Missing correlations in the prediabetes subgroups may be due to the small number of patients.

\section{DISCUSSION}

To our knowledge, this study is the first to show that the sciatic nerve's FA as a surrogate marker for nerve integrity correlates both with electrophysiological parameters of nerves in the distal lower limb and parameters of hand function in patients with diabetes. Also, this study is the first to show correlations between the sciatic nerve's FA and parameters of hand function in patients with prediabetes. Both results are of importance with regards to understanding the course of nerve damage in diabetic neuropathy: despite the assumption that nerve damage in DN parallels clinical symptoms and progresses from distally to proximally starting at the level of the feet and progressing further onward to the level of the hands at later stages, our results indicate that neuropathic changes at the level of the upper limbs parallel those at the level of the lower limbs.

The finding that an association between the sciatic nerve's FA and functional parameters of the hands and the lower leg can already be displayed in patients with prediabetes suggests that functional impairment of the upper extremities occurs during the development of pathological glucose tolerance that later progresses into type 2 diabetes (American Diabetes Association, 2017). This is of importance for the understanding of the progression of neuropathic changes leading to diabetic neuropathy, since these findings suggest that structural nerve damage related to functional impairment occurs prior to or at very early stages of type 2 diabetes and is not, as mostly assumed, a late complication of this disease (Jende et al., 2018, 2020c). The finding that the proximally located sciatic nerve's FA correlates with functional parameters of the distal upper and lower limbs further indicates that the deterioration of nerve microstructure does not progress from distally to further proximally but rather suggests that the entire peripheral nervous system is already affected at the very beginning of DN. This is in line with results from previous studies on MRN in patients with and without DN that have found a proximal predominance of nerve lesions in DN in T2-weighted imaging (Jende et al., 2018, 2020b).

Since studies on MRN have come to show that nerve damage predominates at a proximal level in various neuropathies, the finding that the sciatic nerve's FA codifies functional parameters 


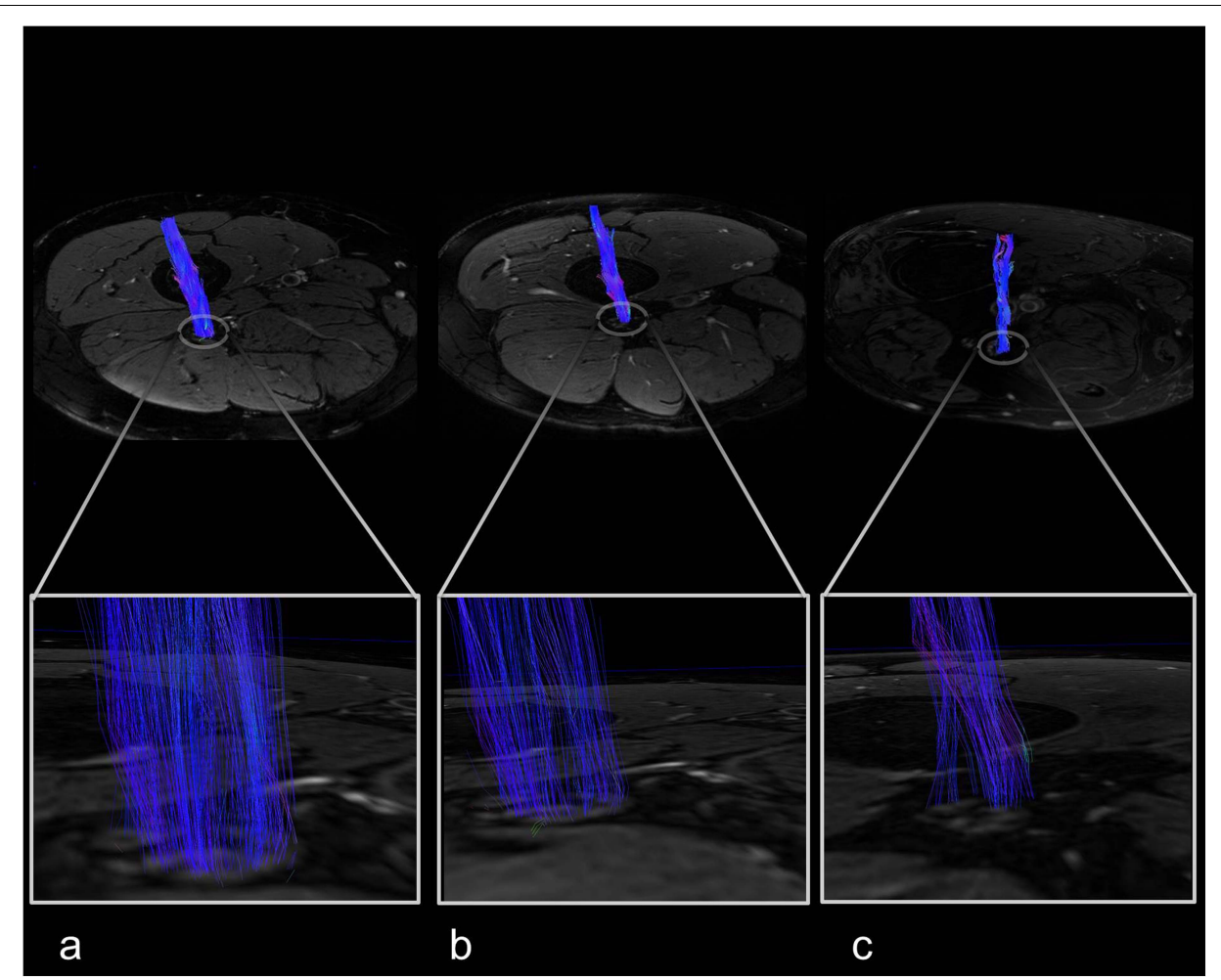

FIGURE 3 | Sciatic nerve fiber tracts with magnified views. (a) Female control, 67 years, neuropathy disability score (NDS) = 0. (b) Female prediabetes patient, 57 years, NDS $=3$. (c) Male type 2 diabetes patient, 58 years, NDS $=7$.

of the upper limb not just in patients with prediabetes and diabetes but also in controls without impaired glucose tolerance, may be of relevance not just for the course of nerve damage in $\mathrm{DN}$ but also other diseases of the central and peripheral nervous system (Jende et al., 2017). Additionally, our findings suggest that FA values represent an objective parameter for assessing the structural integrity of the entire peripheral nervous system in longitudinal clinical studies or interventional studies in patients with DN. One may of curse argue that, since there was a correlation between participants' age and the sciatic nerve's FA in controls and patients with diabetes, the results of this study only display the well-established effect of aging on the structural and functional integrity of the peripheral nervous system (Kronlage et al., 2018). It should be considered, however, that in patients with prediabetes there was no correlation between age and FA whereas correlations between FA and parameters of hand function were significant.

The finding that there is no correlation between FA and patients' age in prediabetes patients but a positive correlation between FA and the scores of the Pegboard test can be explained by the fact that the performance in the Purdue Pegboard test is not just a matter of peripheral nerve function but also depends on cognitive and coordinative skills, which are known to decline with age (Geffe et al., 2016). The finding that there is a correlation of the Pegboard test's results with structural nerve integrity represented by the sciatic nerve's FA indicates that a reduction of the latter is associated with a reduced performance in the
Pegboard test and that, therefore, the structural integrity of peripheral nerves is of critical relevance for the performance in coordinative tasks. The finding that a reduction in FA is not correlated with age in the prediabetes group indicates that, other than cognitive and motor skills at the level of the central nervous system, diabetes-related structural damage to peripheral nerves occurs at very early stages of this disorder. This hypothesis is further supported by the finding that, while participant groups were matched for age, FA was significantly lower in diabetes patients when compared to controls. This indicates that, in addition to the process of aging, metabolic changes associated with prediabetes and diabetes cause auxiliary structural damage to peripheral nerves (Groener et al., 2020). However, it should be noted further that correlations between FA and Pegboard test parameters were significantly weaker for patients in the prediabetes group than for diabetes patients or the control group. Similarly, there are no significant correlations in the prediabetes group between FA and sural SNAP parameters or age, while there are in the diabetes and control group. In order to determine the exact impact of age and metabolic factors on nerve damage in prediabetes more closely, longitudinal studies with larger cohorts of prediabetes patients are required.

One may also argue that this study did not differentiate between type 1 diabetes and type 2 diabetes patients, although previous studies have shown that structural nerve damage differs between diabetes types for T2-weighted imaging (Jende et al., 2018). For DTI imaging, however, FA was found as a reliable 
TABLE 2 | Correlations of the sciatic nerve's FA with clinical, epidemiological, and serological data of diabetes patients, prediabetes patients, and controls.

\begin{tabular}{|c|c|c|c|c|c|c|}
\hline & \multicolumn{2}{|c|}{ FA diabetes patients } & \multicolumn{2}{|c|}{ FA prediabetes patients } & \multicolumn{2}{|c|}{ FA controls } \\
\hline & $r$ & $p$ & $r$ & $p$ & $r$ & $p$ \\
\hline Age (years) & -0.48 & $<0.001$ & 0.44 & 0.202 & -0.63 & 0.001 \\
\hline Body mass index $\left(\mathrm{kg} / \mathrm{m}^{2}\right)$ & -0.18 & 0.140 & -0.08 & 0.832 & 0.03 & 0.881 \\
\hline NDS & -0.53 & $<0.001$ & -0.58 & 0.082 & -0.47 & 0.017 \\
\hline NSS & -0.20 & 0.106 & -0.57 & 0.107 & -0.36 & 0.107 \\
\hline HbA1c (\%) & 0.15 & 0.211 & -0.07 & 0.844 & -0.42 & 0.039 \\
\hline Cystatin C (mg/l) & -0.45 & $<0.001$ & -0.27 & 0.489 & -0.22 & 0.320 \\
\hline Glomerular filtration rate (ml/min) & 0.46 & $<0.001$ & 0.21 & 0.571 & 0.23 & 0.291 \\
\hline Triglycerides (mg/dl) & 0.05 & 0.694 & 0.06 & 0.876 & -0.04 & 0.867 \\
\hline Total serum cholesterol (mg/dl) & 0.17 & 0.151 & -0.10 & 0.776 & -0.15 & 0.462 \\
\hline HDL cholesterol (mg/dl) & 0.17 & 0.157 & -0.11 & 0.762 & 0.07 & 0.728 \\
\hline LDL cholesterol (mg/dl) & 0.10 & 0.414 & 0.05 & 0.770 & -0.18 & 0.396 \\
\hline Sural $N C V(m / s)$ & 0.24 & 0.128 & -0.28 & 0.505 & -0.12 & 0.568 \\
\hline Sural SNAP $(\mu V)$ & 0.47 & 0.006 & 0.30 & 0.471 & 0.58 & 0.003 \\
\hline Tibial NCV (m/s) & 0.47 & $<0.001$ & 0.61 & 0.081 & 0.35 & 0.099 \\
\hline Tibial CMAP $(\mu \mathrm{V})$ & 0.62 & $<0.001$ & 0.61 & 0.081 & 0.30 & 0.150 \\
\hline Peroneal NCV (m/s) & 0.52 & $<0.001$ & 0.43 & 0.246 & 0.31 & 0.142 \\
\hline Peroneal CMAP $(\mu \mathrm{V})$ & 0.62 & $<0.001$ & 0.51 & 0.160 & 0.18 & 0.411 \\
\hline Total Pegboard Test score & 0.52 & $<0.001$ & 0.79 & 0.007 & 0.76 & $<0.001$ \\
\hline Pegboard Test of dominant hand & 0.61 & $<0.001$ & 0.65 & 0.044 & 0.64 & 0.001 \\
\hline Pegboard Test of non-dominant hand & 0.43 & $<0.001$ & 0.71 & 0.022 & 0.55 & 0.005 \\
\hline Pegboard Test of both hands & 0.38 & 0.002 & 0.70 & 0.024 & 0.59 & 0.002 \\
\hline Pegboard Assembly Test & 0.46 & $<0.001$ & 0.71 & 0.022 & 0.61 & 0.001 \\
\hline
\end{tabular}

NDS, Neuropathy Disability Score; NSS, Neuropathy Severity Score; HbA1c, glycated hemoglobin; GFR, glomerular filtration rate; HDL, high density lipoprotein; LDL,

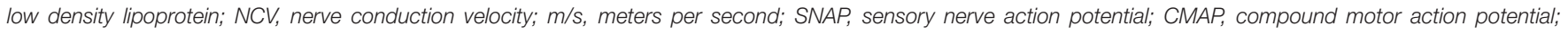
$\mu \mathrm{V}$, microvolt.

marker for structural nerve integrity at the level of the lower limbs in both type 1 and type 2 diabetes (Vaeggemose et al., 2017a, 2020).

Our study is limited by the fact that only cross-sectional data were used, which does not allow for conclusions on the predictive value of the sciatic nerve's FA. Also, the group of prediabetes patients was too small to allow for exact conclusions of the interaction between aging and metabolic factors. Another limitation is that we were only testing motor function and coordination of the upper limbs but did not test for sensory gain or loss.

While, usually, improved tractography methods to select multi fiber tracts in cerebral imaging can be challenging due to the difficulty of selecting adequate regions of interest for tract seeding (Wilkins et al., 2015; Christidi et al., 2016), the VOI selection in sciatic nerve processing is straight-forward due to the nature of peripheral nerves that can be easily identified on T2w images. Nordic BrainEx was shown to agree in major fiber bundle reconstruction with other major software vendors such as DSI Studio or Philips FiberTrak, while there were differences for smaller fiber bundles (Christidi et al., 2016). It should therefore be noted that systematic errors during the automated reconstruction process may impact the DTI parameter results, however, the FACT algorithm used in Nordic BRAINEX is a commonly used DTI reconstruction algorithm, and seeding errors are supposedly low in lieu of the above considerations (Mukherjee et al., 2008).
Further potential sources of error are the FA cutoff, which we chose in agreement with previous publications on peripheral nerve DTI analyses (Kwon et al., 2015; Oudeman et al., 2020), partial volume effects, and, naturally, experimental parameters such as resolution and signal-to-noise ratios (Okamoto et al., 2010; Barrio-Arranz et al., 2015). We chose a comparatively high DTI in-plane resolution, see e.g. (van Steenkiste et al., 2016), to reduce DTI processing errors, as well as multiple averages to reduce signal-to-noise ratio. Considering that sciatic nerve fat fractions are elevated in patients with increased BMI, a missing correlation of sciatic nerve FA with BMI (Table 2) indicates that there is no significant impact of intra- or perineural fat on FA calculation.

One further limitation may be the spatial heterogeneity of the gradients (Krzyżak and Olejniczak, 2015; Borkowski and Krzyżak, 2019). Taken together, these types of errors are likely averaged for a larger number of patients, as in the diabetes and control group.

In summary, this study is the first to show that the sciatic nerve's FA is a surrogate marker for nerve function of the distal upper and lower limbs in patients with diabetes and prediabetes. Our findings suggest that proximal nerve damage in diabetes parallels distal nerve function even before patients start to experience clinical symptoms, which may also be relevant with regard to PNS damage in other neuropathies. Further longitudinal studies on the predictive value of the sciatic nerve's 

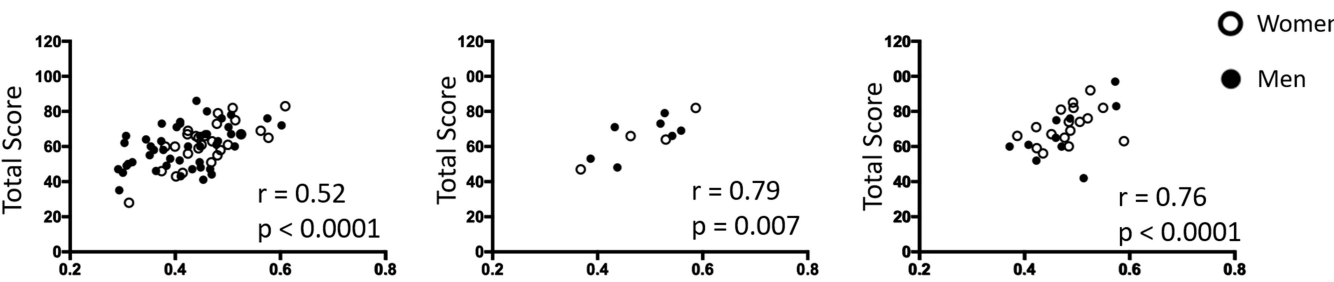

A Sciatic nerve FA, Diabetes

B Sciatic nerve FA, Prediabetes

C Sciatic nerve FA, Controls
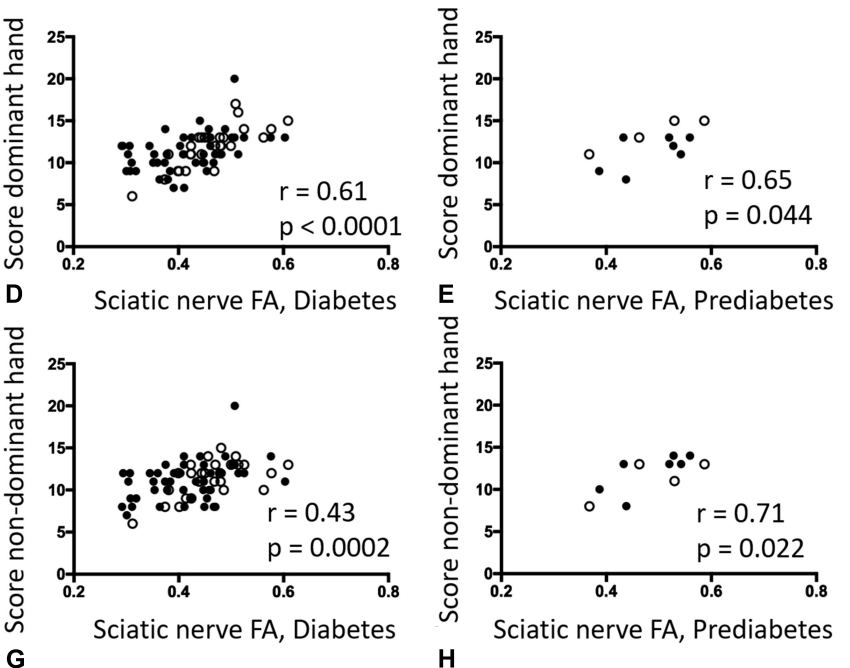

E Sciatic nerve FA, Prediabetes
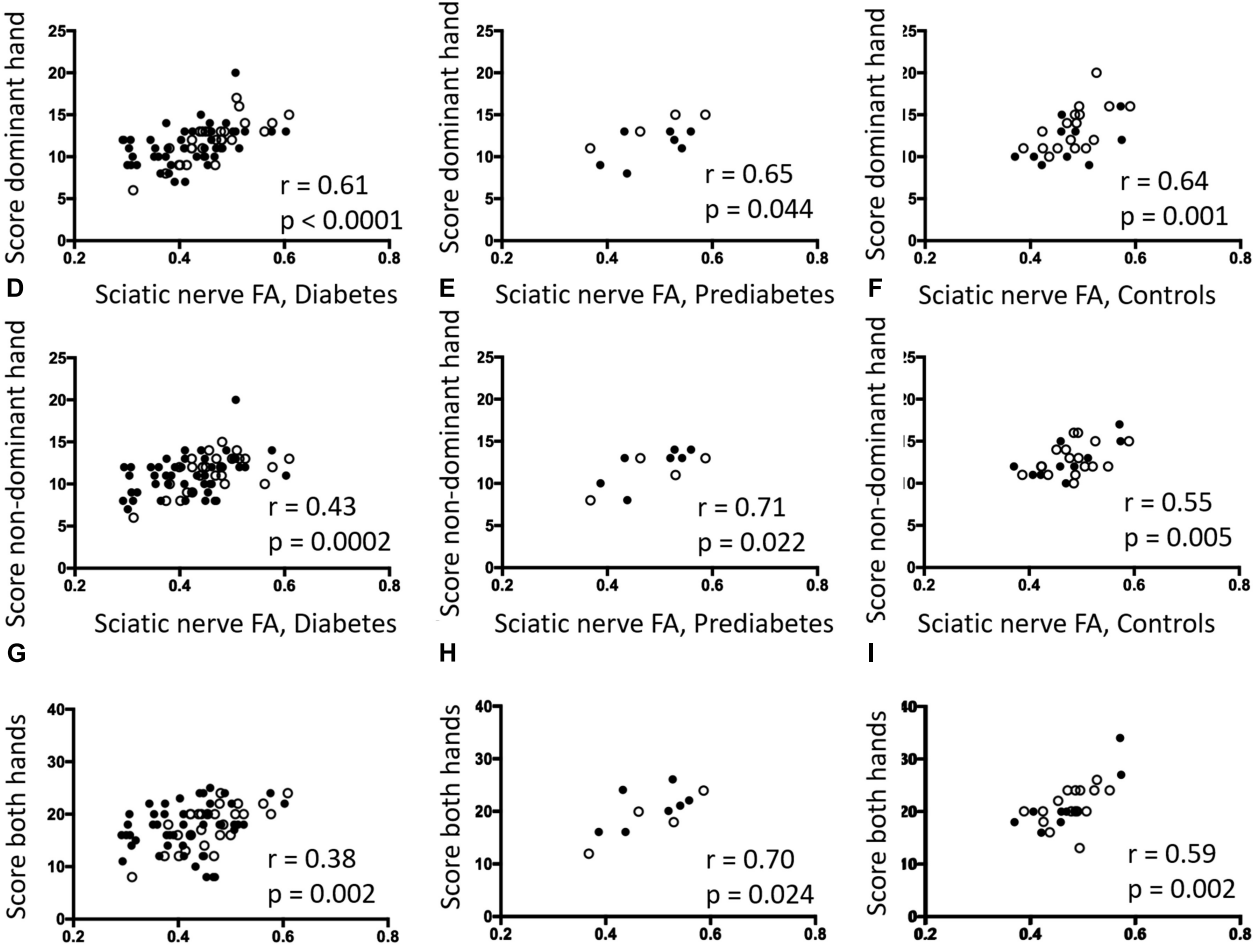

J Sciatic nerve FA, Diabetes

K Sciatic nerve FA, Prediabetes L

Sciatic nerve FA, Controls
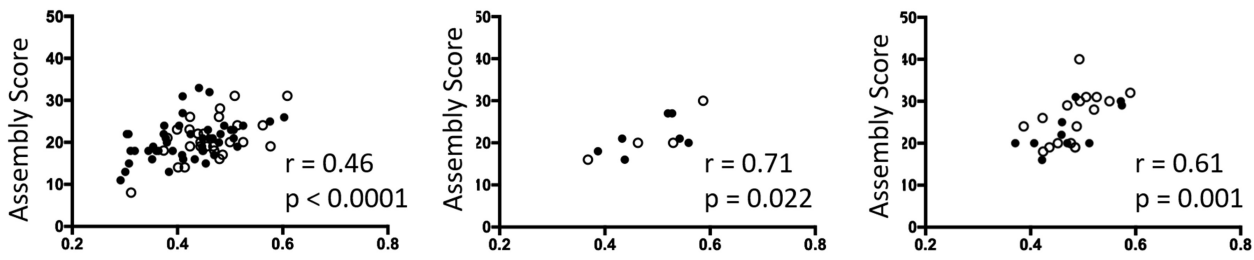

M Sciatic nerve FA, Diabetes

N Sciatic nerve FA, Prediabetes

- Sciatic nerve FA, Controls

FIGURE 4 | Correlative statistics of Purdue Pegboard Test scores and sciatic nerve fractional anisotropy. (A) Correlation of the sciatic nerve's fractional anisotropy (FA) in patients with diabetes with the total Purdue Pegboard Test score $(r=0.52 ; p<0.001 ; 95 \%$ confidence interval $(C l)=0.31$ to 0.67$)$. (B) Correlation of the sciatic nerve's FA in patients with prediabetes with the total Purdue Pegboard Test score $(r=0.79 ; p=0.007 ; 95 \% \mathrm{Cl}=0.31$ to 0.95$)$. (C) Correlation of the sciatic nerve's FA in controls with the total Purdue Pegboard Test score $(r=0.76 ; p<0.001 ; 95 \% \mathrm{Cl}=0.52$ to 0.89$)$. (D) Correlation of the sciatic nerve's FA in patients with diabetes with the Purdue Pegboard Test score of the dominant hand $(r=0.61 ; p<0.001 ; 95 \% \mathrm{Cl}=0.43$ to 0.74 ). (E) Correlation of the sciatic nerve's FA in patients with prediabetes with the Purdue Pegboard Test score of the dominant hand $(r=0.65 ; p=0.044 ; 95 \% \mathrm{Cl}=0.52$ to 0.90$)$. (F) Correlation of the sciatic nerve's FA in controls with the Purdue Pegboard Test score of the dominant hand $(r=0.64 ; p=0.001 ; 95 \% \mathrm{Cl}=0.22$ to 0.77$)$. (G) Correlation of the sciatic nerve's FA in patients with diabetes with the Purdue Pegboard Test score of the non-dominant hand $(r=0.43 ; p=0.0002 ; 95 \% \mathrm{Cl}=0.22$ to 0.60$)$. (H) Correlation of the sciatic nerve's FA in patients with prediabetes with the Purdue Pegboard Test score of the non-dominant hand $(r=0.71 ; p=0.022 ; 95 \% \mathrm{Cl}=0.21$ to 0.92$)$. (I) Correlation of the sciatic nerve's FA in controls with the Purdue Pegboard Test score of the non-dominant hand $(r=0.55 ; p=0.005 ; 95 \% \mathrm{Cl}=0.19$ to 0.76$)$. (J) Correlation of the sciatic nerve's FA in patients with diabetes with the Purdue Pegboard Test score of both hands $(r=0.38 ; p=0.002 ; \mathrm{C} 95 \% \mathrm{Cl}=0.14$ to 0.54$)$. (K) Correlation of the sciatic nerve's FA in patients with prediabetes with the Purdue Pegboard Test score of both hands $(r=0.70 ; p=0.024 ; 95 \% \mathrm{Cl}=0.16$ to 0.91$)$. (L) Correlation of the sciatic nerve's FA in controls with the Purdue Pegboard Test score of both hands $(r=0.59 ; p=0.002 ; 95 \% \mathrm{Cl}=0.37$ to 0.84 ). (M) Correlation of the sciatic nerve's FA in patients with diabetes with the Purdue Pegboard Test assembly score $(r=0.46 ; p<0.001 ; 95 \% \mathrm{Cl}=0.26$ to 0.63$)$. (N) Correlation of the sciatic nerve's FA in patients with prediabetes with the Purdue Pegboard Test assembly score ( $r=0.71 ; p=0.022 ; 95 \% \mathrm{Cl}=0.20$ to 0.92 ). (0) Correlation of the sciatic nerve's FA in controls with the Purdue Pegboard Test assembly score $(r=0.61 ; p=0.001 ; 95 \% \mathrm{Cl}=0.26$ to 0.79$)$. 
FA for disease progression in $\mathrm{DN}$ and other neuropathies are warranted.

\section{DATA AVAILABILITY STATEMENT}

The datasets presented in this article are not readily available because they contain sensitive patient information. The data supporting the conclusions of this article will be made available upon reasonable request by any qualified researcher. Requests to access the datasets should be directed to FK, felix.kurz@med.uniheidelberg.de.

\section{ETHICS STATEMENT}

The studies involving human participants were reviewed and approved by the Ethics Committee of the Medical Faculty of the University of Heidelberg. The patients/participants provided their written informed consent to participate in this study.

\section{AUTHOR CONTRIBUTIONS}

JJ, MB, SH, PN, and FK designed and coordinated the study. JJ, CM, JG, JK, AJ, and FK contributed to the organization of participants. JJ, AJ, and FK collected MR data. AH and FK developed image analysis tools. ZK, LA-R, JG, and SK collected clinical, serological, and electrophysiological data. JJ and FK analyzed the data, wrote the manuscript with input from all coauthors.

\section{FUNDING}

The German research council (DFG, SFB 1158) and the international Foundation for Research in paraplegia (IRP) provided financial support for personnel expenditure, MR imaging costs and costs for the technical equipment required for electrophysiological and serological analysis. The DFG and the IRP had no influence on the study design, collection and

\section{REFERENCES}

American Diabetes Association (2017). 2. Classification and diagnosis of diabetes. Diabetes Care 40(Suppl. 1), S11-S24. doi: 10.2337/dc17-S005

Barrio-Arranz, G., de Luis-García, R., Tristán-Vega, A., Martín-Fernández, M., and Aja-Fernández, S. (2015). Impact of MR acquisition parameters on DTI scalar indexes: a tractography based approach. PLoS One 10:e0137905. doi: 10.1371/journal.pone.0137905

Borkowski, K., and Krzyżak, A. T. (2019). Assessment of the systematic errors caused by diffusion gradient inhomogeneity in DTI-computer simulations. NMR Biomed. 32:e4130. doi: 10.1002/nbm.4130

Christidi, F., Karavasilis, E., Samiotis, K., Bisdas, S., and Papanikolaou, N. (2016). Fiber tracking: a qualitative and quantitative comparison between four different software tools on the reconstruction of major white matter tracts. Eur. J. Radiol. Open 3, 153-161. doi: 10.1016/J.EJRO.2016. 06.002 analysis of data or on the writing of the article. JJ received grants from the German Research Foundation (SFB 1158) and from the International Foundation for Research in Paraplegia. ZK received grants from the Deutsches Zentrum für Diabetesforschung (DZD) e.V. CM reports no disclosures. JG received grants from the Deutsches Zentrum für Diabetesforschung (DZD) e.V. LA-R reports no disclosures. JK received research grants, personal fees, and lecture honoraria from Alnylam Pharmaceutical (MA, United States), the Olympia Morata stipend grant from the Medical Faculty of the University of Heidelberg, lecture honoraria and financial support for conference attendance from Pfizer, and advises for Akcea Therapeutics. AJ received a grant from the Dietmar Hopp foundation (grant No. 23011228). $\mathrm{AH}$ received grants from the German Research Foundation (DFG grant KU 3555/1-1). SH received a grant from the Dietmar Hopp foundation (grant No. 23011228) and the German Research Council (DFG, SFB 1118). PN received grants from the German research Council (DFG, SFB 1118 and 1158) and from Novo Nordisk. MB received grants and personal fees from Codman, Guerbet, Bayer and Novartis, personal fees from Roche, Teva, Springer, Boehringer, Grifols, Braun and grants from the European Union, Siemens, the Dietmar Hopp foundation, Stryker and the German Research Council (DFG, SFB 1118 and 1158). SK reports no disclosures. FK was supported by the German Research Foundation (KU 3555/1-1) and the HoffmannKlose foundation of Heidelberg University Hospital.

\section{ACKNOWLEDGMENTS}

We thank Mrs. Dorothea Willich (Department of Neuroradiology, Heidelberg University Hospital) for her ongoing support and excellent technical performance of all MRN examinations.

\section{SUPPLEMENTARY MATERIAL}

The Supplementary Material for this article can be found online at: https://www.frontiersin.org/articles/10.3389/fnins. 2021.642589/full\#supplementary-material

Feldman, E. L., Nave, K.-A., Jensen, T. S., and Bennett, D. L. H. (2017). New horizons in diabetic neuropathy: mechanisms, bioenergetics, and pain. Neuron 93, 1296-1313.

Geffe, S., Schindlbeck, K. A., Mehl, A., Jende, J., Klostermann, F., and Marzinzik, F. (2016). The single intake of levodopa modulates implicit learning in drug naïve, de novo patients with idiopathic Parkinson's disease. J. Neural Transm. 123, 601-610. doi: 10.1007/s00702-016-1557-y

Groener, J. B., Jende, J. M. E., Kurz, F. T., Kender, Z., Treede, R.-D., SchuhHofer, S., et al. (2020). Understanding diabetic neuropathy-from subclinical nerve lesions to severe nerve fiber deficits: a cross-sectional study in patients with type 2 diabetes and healthy control subjects. Diabetes 69, 436-447. doi: 10.2337/db19-0197

Jende, J. M. E., Groener, J. B., Kender, Z., Hahn, A., Morgenstern, J., Heiland, S., et al. (2020a). Troponin T parallels structural nerve damage in type 2 diabetes: a cross-sectional study using magnetic resonance neurography. Diabetes 69, 713-723. doi: 10.2337/db19-1094 
Jende, J. M. E., Groener, J. B., Kender, Z., Rother, C., Hahn, A., Hilgenfeld, T., et al. (2020b). Structural nerve remodeling at 3-T MR neurography differs between painful and painless diabetic polyneuropathy in type 1 or 2 diabetes. Radiology 294, 405-414. doi: 10.1148/radiol.2019191347

Jende, J. M. E., Groener, J. B., Oikonomou, D., Heiland, S., Kopf, S., Pham, M., et al. (2018). Diabetic neuropathy differs between type 1 and type 2 diabetes: insights from magnetic resonance neurography. Ann. Neurol. 83, 588-598.

Jende, J. M. E., Groener, J. B., Rother, C., Kender, Z., Hahn, A., Hilgenfeld, T., et al. (2019). Association of serum cholesterol levels with peripheral nerve damage in patients with type 2 diabetes. JAMA Netw. Open 2:e194798.

Jende, J. M. E., Hauck, G. H., Diem, R., Weiler, M., Heiland, S., Wildemann, B., et al. (2017). Peripheral nerve involvement in multiple sclerosis: demonstration by magnetic resonance neurography. Ann. Neurol. 82, 676-685.

Jende, J. M. E., Kender, Z., Rother, C., Alvarez-Ramos, L., Groener, J. B., Pham, M., et al. (2020c). Diabetic polyneuropathy is associated with pathomorphological changes in human dorsal root ganglia: a study using 3T MR neurography. Front. Neurosci. 14:570744. doi: 10.3389/fnins.2020.570744

Kopf, S., Groener, J. B., Kender, Z., Fleming, T., Bischoff, S., Jende, J., et al. (2018a). Deep phenotyping neuropathy: an underestimated complication in patients with pre-diabetes and type 2 diabetes associated with albuminuria. Diabetes Res. Clin. Pract. 146, 191-201. doi: 10.1016/j.diabres.2018. 10.020

Kopf, S., Groener, J. B., Kender, Z., Fleming, T., Brune, M., Riedinger, C., et al. (2018b). Breathlessness and restrictive lung disease: an important diabetesrelated feature in patients with type 2 diabetes. Respiration 96, 29-40. doi: 10.1159/000488909

Kronlage, M., Schwehr, V., Schwarz, D., Godel, T., Uhlmann, L., Heiland, S., et al. (2018). Peripheral nerve diffusion tensor imaging (DTI): normal values and demographic determinants in a cohort of 60 healthy individuals. Eur. Radiol. 28, 1801-1808. doi: 10.1007/s00330-017-5134-z

Krzyżak, A. T., and Olejniczak, Z. (2015). Improving the accuracy of PGSE DTI experiments using the spatial distribution of $\mathrm{b}$ matrix. Magn. Reson. Imaging 33, 286-295. doi: 10.1016/j.mri.2014.10.007

Kwon, B. C., Koh, S. H., and Hwang, S. Y. (2015). Optimal parameters and location for diffusion-tensor imaging in the diagnosis of carpal tunnel syndrome: a prospective matched case-control study. AJR Am. J. Roentgenol. 204, 12481254. doi: 10.2214/AJR.14.13371

Mori, S., Crain, B. J., Chacko, V. P., and van Zijl, P. C. (1999). Three-dimensional tracking of axonal projections in the brain by magnetic resonance imaging. Ann. Neurol. 45, 265-269. doi: 10.1002/1531-8249(199902)45:2<265::aid-ana21<3.0. co;2-3

Mukherjee, P., Berman, J. I., Chung, S. W., Hess, C. P., and Henry, R. G. (2008). Diffusion tensor MR imaging and fiber tractography: theoretic underpinnings. Am. J. Neuroradiol. 29, 632-641. doi: 10.3174/ajnr.A1051

Nawroth, P. P., Bendszus, M., Pham, M., Jende, J., Heiland, S., Ries, S., et al. (2018). The quest for more research on painful diabetic neuropathy. Neuroscience 387, 28-37. doi: 10.1016/j.neuroscience.2017.09.023

NordicNeuroLab AS (2019). nordic BrainEX Tutorial - DTI Module. Available online at: http://downloads.nordicneurolab.com/SW/nBX/Tutorials/Tutorial\% 20-\%20DTI.pdf (accessed January 31, 2021).

O’Donnell, L. J., Suter, Y., Rigolo, L., Kahali, P., Zhang, F., Norton, I., et al. (2017). Automated white matter fiber tract identification in patients with brain tumors. Neuroimage Clin. 13, 138-153. doi: 10.1016/j.nicl.2016.11.023
Okamoto, Y., Kunimatsu, A., Kono, T., Nasu, K., Sonobe, J., and Minami, M. (2010). Changes in MR diffusion properties during active muscle contraction in the calf. Magn. Reson. Med. Sci. 9, 1-8. doi: 10.2463/mrms.9.1

Oudeman, J., Eftimov, F., Strijkers, G. J., Schneiders, J. J., Roosendaal, S. D., Engbersen, M. P., et al. (2020). Diagnostic accuracy of MRI and ultrasound in chronic immune-mediated neuropathies. Neurology 94, e62-e74. doi: 10.1212/ WNL.0000000000008697

Symonds, T., Campbell, P., and Randall, J. A. (2017). A review of muscle- and performance-based assessment instruments in DM1. Muscle Nerve 56, 78-85. doi: $10.1002 /$ mus. 25468

Tesfaye, S., Chaturvedi, N., Eaton, S. E. M., Ward, J. D., Manes, C., IonescuTirgoviste, C., et al. (2005). Vascular risk factors and diabetic neuropathy. N. Engl. J. Med. 352, 341-350. doi: 10.1056/NEJMoa032782

Vaeggemose, M., Haakma, W., Pham, M., Ringgaard, S., Tankisi, H., Ejskjaer, N., et al. (2020). Diffusion tensor imaging MR neurography detects polyneuropathy in type 2 diabetes. J. Diabetes Complications 34:107439. doi: 10.1016/j.jdiacomp. 2019.107439

Vaeggemose, M., Pham, M., Ringgaard, S., Tankisi, H., Ejskjaer, N., Heiland, S., et al. (2017a). Diffusion tensor imaging MR neurography for the detection of polyneuropathy in type 1 diabetes. J. Magn. Reson. Imaging 45, 1125-1134. doi: $10.1002 /$ jmri. 25415

Vaeggemose, M., Pham, M., Ringgaard, S., Tankisi, H., Ejskjaer, N., Heiland, S., et al. (2017b). Magnetic resonance neurography visualizes abnormalities in sciatic and tibial nerves in patients with type 1 diabetes and neuropathy. Diabetes 66, 1779-1788. doi: 10.2337/db16-1049

van Steenkiste, G., Jeurissen, B., Baete, S., den Dekker, A. J., Poot, D. H. J., Boada, F., et al. (2016). High Resolution Diffusion Tensor Reconstruction from Simultaneous Multi-Slice Acquisitions in a Clinically Feasible Scan time. Proceedings of the International Society for Magnetic Resonance in Medicine 0002. Available online at: http://ismrm.gitlab.io/2016/0002.html (Accessed February 3, 2021).

Wilkins, B., Lee, N., Gajawelli, N., Law, M., and Leporé, N. (2015). Fiber estimation and tractography in diffusion MRI: development of simulated brain images and comparison of multi-fiber analysis methods at clinical b-values. Neuroimage 109, 341-356. doi: 10.1016/j.neuroimage.2014.12.060

Yang, C.-J., Hsu, H.-Y., Lu, C.-H., Chao, Y.-L., Chiu, H.-Y., and Kuo, L.C. (2018). Do we underestimate influences of diabetic mononeuropathy or polyneuropathy on hand functional performance and life quality? J. Diabetes Investig. 9, 179-185. doi: 10.1111/jdi.12649

Young, M. J., Boulton, A. J., MacLeod, A. F., Williams, D. R., and Sonksen, P. H. (1993). A multicentre study of the prevalence of diabetic peripheral neuropathy in the United Kingdom hospital clinic population. Diabetologia 36, 150-154. doi: $10.1007 / \mathrm{BF} 00400697$

Conflict of Interest: The authors declare that the research was conducted in the absence of any commercial or financial relationships that could be construed as a potential conflict of interest.

Copyright (c) 2021 Jende, Kender, Mooshage, Groener, Alvarez-Ramos, Kollmer, Juerchott, Hahn, Heiland, Nawroth, Bendszus, Kopf and Kurz. This is an open-access article distributed under the terms of the Creative Commons Attribution License (CC BY). The use, distribution or reproduction in other forums is permitted, provided the original author(s) and the copyright owner(s) are credited and that the original publication in this journal is cited, in accordance with accepted academic practice. No use, distribution or reproduction is permitted which does not comply with these terms. 